\title{
HIGH ENERGETIC GAMMA RAY BURSTS AND THEIR SPECTRAL PROPERTIES WITHIN THE FIRESHELL MODEL
}

\author{
B. PATRICELLI,,${ }^{1,2,3}$ M.G. BERNARDini, ${ }^{1,2,4}$ C.L. BIANCO,${ }^{1,2}$ L.CAITO,${ }^{1,2}$ \\ G.DE BARROS ${ }^{1,2}$ L. IZZO, ${ }^{1,2}$ R. RUFFINI,${ }^{1,2,5}$ G. VERESHCHAGIN ${ }^{1}$ \\ ${ }^{1}$ ICRANet and ICRA, Piazzale della Repubblica 10, I-65122 Pescara, Italy \\ 2 Dipartimento di Fisica, Università di Roma "Sapienza", Piazzale Aldo Moro 5, I-00185 \\ Roma, Italy \\ ${ }^{3}$ LAPTH, 9, Chemin de Bellevue - BP 110, 74941, Annecy-le-Vieux Cedex, France \\ ${ }^{4}$ INAF - Osservatorio Astronomico di Brera, via Emilio Bianchi 46, I-23807 Merate (Lc), Italy \\ ${ }^{5}$ ICRANet, Université de Nice "Sophia Antipolis", Grand Château, 28, avenue de Valrose - BP \\ 2135, 06103 Nice Cedex 2, France \\ E-mails: \\ barbara.patricelli@icranet.org,maria.bernardini@icra.it,bianco@icra.it,letizia.caito@icra.it, \\ guga.icra@gmail.com,luca.izzo@icra.it,ruffini@icra.it,veresh@icra.it.
}

\begin{abstract}
The analysis of various Gamma Ray Bursts (GRBs) characterized by an isotropic energy $E_{\text {iso }} \lesssim 10^{53}$ ergs within the fireshell model has shown how that the observed N(E) spectrum of their prompt emission can be reproduced in a satisfactory way by assuming a thermal spectrum in the comoving frame of the fireshell. Nevertheless, from the study of higher energetic bursts $\left(E_{i s o} \gtrsim 10^{54} \mathrm{ergs}\right)$ such as, for example, GRB 080319B, some discrepancies between the numerical simulations and the observational data have been observed. We investigate a different spectrum of photons in the comoving frame of the fireshell in order to better reproduce the spectral properties of GRB prompt emission within the fireshell model. We introduce a phenomenologically modified comoving thermal spectrum: a spectrum characterized by a different asymptotic low energy slope with respect to the thermal one. We test this spectrum by comparing the numerical simulations with the observed prompt emission spectra of various GRBs; we present, as an exaple, the case of GRB 080319B.
\end{abstract}

Keywords: Gamma-ray bursts; gamma-ray sources; gamma-rays.

\section{Introduction}

Over the years many efforts have been made to explain the observational properties of GRBs and this has lead to the development of different theoretical models such as, for example, the fireball model ${ }^{1,2}$, the electromagnetic model ${ }^{3,4}$ and the photospheric model ${ }^{5,6,7,8}$. Another model, which presents a very different approach with respect to the others, is the fireshell model. One of the main features of the fireshell model is that it analyses the GRB phenomenon as a whole: in fact, the observational properties of GRBs are reproduced starting from the initial conditions characterizing the progenitor; the dynamics of the system is uniquely fixed 
since its creation ${ }^{9,10,11,12}$ and the solutions of the equations of motion of the system $^{13,14}$ are calculated without the approximations usually assumed in the fireball model, in which the self-similar solution of Blandford \& McKee ${ }^{15}$ is adopted (see, for example, Ref. 16, 17, 18, 19, 20, 21, 22, 23, 24, 25, 26). Another important point is that the GRB light curves and spectra are obtained by assuming a spectral energy distribution of the photons in the comoving frame of the fireshell: this approach represents an attempt to identify the underlying physical process responsible for the energy emission. Each observed instantaneous spectrum is the convolution over the corresponding EquiTemporal Surface (EQTS) ${ }^{13,14}$ of thousands of comoving spectra, duly weighted by their corresponding Lorentz and Doppler factors. Time integrated spectra are then obtained by integrating over the observation time ${ }^{27}$. For simplicity, a comoving thermal spectrum was assumed ${ }^{28}$ and in this way it has been possible to interpret successfully the observational properties of many GRBs characterized by an isotropic energy $E_{\text {iso }} \lesssim 10^{53}$ ergs such as, for example, GRB 031203 (see Ref. 27). Nevertheless, from the study of higher energetic bursts $\left(E_{\text {iso }} \gtrsim 10^{54}\right.$ ergs) such as, for example, GRB 080319B some discrepancies between the numerical simulations and the prompt emission spectra have been observed. In this work we investigate a different spectral energy distribution of photons in the comoving frame of the fireshell in order to better reproduce the observational data of GRBs.

\section{The GRB luminosity and spectrum within the fireshell model}

Within the fireshell model, the GRB luminosity at the detector arrival time $t_{a}^{d}$ per unit solid angle $d \Omega$ and in the energy band $\left[\nu_{1}, \nu_{2}\right]$ is given by ${ }^{11}$

$$
\frac{d E^{\left[\nu_{1}, \nu_{2}\right]}}{d t_{a}^{d} d \Omega}=\int_{E Q T S} \frac{\Delta \epsilon}{4 \pi} v \cos \theta \Lambda^{4} \frac{d t}{d t_{a}^{d}} W\left(\nu_{1}, \nu_{2}, T_{a r r}\right) d \Sigma,
$$

where $\Delta \epsilon=\frac{\Delta E_{i n t}}{V}$ is the emitted energy density released in the interaction of the accelerated baryons with the CBM measured in the comoving frame, $\Lambda=$ $\{\gamma[1-(v / c) \cos \theta]\}^{-1}$ is the Doppler factor, $\mathrm{W}\left(\nu_{1}, \nu_{2}, T_{a r r}\right)$ is an "effective weight" required to evaluate only the contributions in the energy band $\left[\nu_{1}, \nu_{2}\right], d \Sigma$ is the surface element of the EQTS at detector arrival time $t_{a}^{d}$ on which the integration is performed and $T_{\text {arr }}$ is the observed temperature of the radiation emitted from $d \Sigma$.

The "effective weight" $\mathrm{W}\left(\nu_{1}, \nu_{2}, T_{\text {arr }}\right)$ is defined as the ratio between the energy density emitted in a given energy band $\left[\nu_{1}, \nu_{2}\right]$ and the bolometric energy density:

$$
W\left(\nu_{1}, \nu_{2}, T_{a r r}\right)=\frac{\int_{\epsilon_{1}}^{\epsilon_{2}}\left(\frac{d N_{\gamma}}{d V d \epsilon}\right) \epsilon d \epsilon}{\int_{0}^{\infty}\left(\frac{d N_{\gamma}}{d V d \epsilon}\right) \epsilon d \epsilon}
$$

where $\frac{d N_{\gamma}}{d V d \epsilon}$ is the number density of photons per unit of energy. We now introduce the new spectral energy distribution for the radiation emitted in the comoving frame of the fireshell: the "modified" thermal spectrum. This spectrum is characterized by a different asymptotic power-law index in the low energy region with respect to 


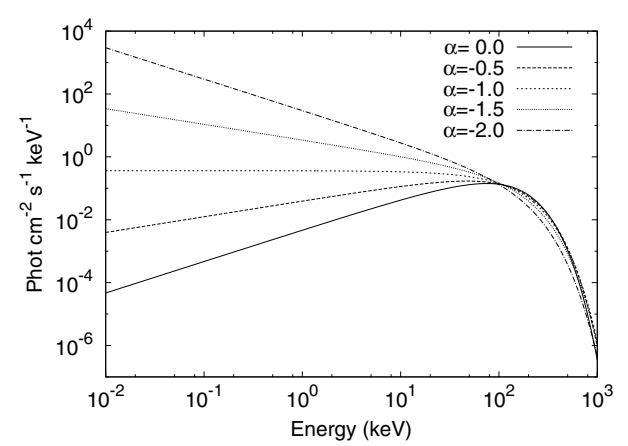

Fig. 1. Theoretically predicted instantaneous spectra characterized by the same temperature and different values of the index $\alpha$. The curve with $\alpha=0.0$ corresponds to the pure thermal spectrum case.

the thermal one. This index is represented by a free parameter $\alpha$, so that the pure thermal spectrum corresponds to the case $\alpha=0$ :

$$
\frac{d N_{\gamma}}{d V d \epsilon}=\left(\frac{8 \pi}{h^{3} c^{3}}\right)\left(\frac{\epsilon}{k_{B} T}\right)^{\alpha} \frac{\epsilon^{2}}{\exp \left(\frac{\epsilon}{k_{B} T}\right)-1},
$$

where $h$ is the Planck constant, $c$ is the speed of light, $k_{B}$ is the Boltzmann constant and $T$ is the temperature.

By using the Eq. (3) and introducing the variable $y=\frac{\epsilon}{k_{B} T}$, we obtain the following expression for the "effective weight":

$$
W\left(\nu_{1}, \nu_{2}, T_{a r r}\right)=\frac{\int_{y_{1}}^{y^{2}} \frac{y^{\alpha+3}}{\exp (y)-1} d y}{\Gamma(4+\alpha) L i_{4+\alpha}(1)},
$$

where $\Gamma(z)=\int_{0}^{\infty} t^{z-1} e^{-t} d t$ is the Gamma function and $L i_{n}(z)=\sum_{k=1}^{\infty} \frac{z^{k}}{k^{n}}$ is the Jonquière's function.

In Fig. 1 there are shown several theoretically predicted instantaneous spectra characterized by the same temperature and different values of the index $\alpha$. It can be seen that the main effect of varying the value of $\alpha$ is a change in the low energy slope of the spectral energy distribution. In particular, by decreasing $\alpha$ the low energy emission increases. Around the peak energy the spectrum is instead only weakly dependent on the value of $\alpha$.

\section{Comparison with the observational data}

We test the "modified" thermal spectrum by comparing the numerical simulations with the observed prompt emission spectra of GRBs. Here we present, as a specific example, the analysis of GRB 080319B. This GRB is a very bright burst: it is characterized by a redshift $z=0.937$ (see Ref. 29) and an isotropic energy release of $E_{\gamma}=1.32 \times 10^{54} \mathrm{ergs}$ (see Ref. 30). Furthermore, it is the most luminous GRB 


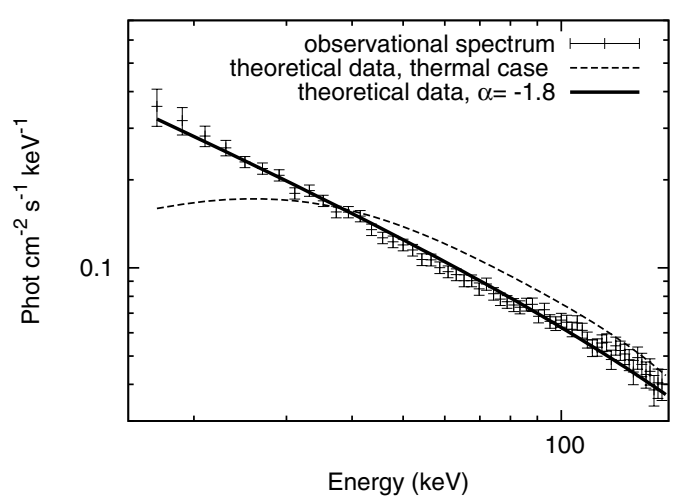

Fig. 2. Theoretically simulated prompt emission spectrum of GRB 080319B integrated over $10 \mathrm{~s}$ with $\alpha=-1.8$ (solid line) and $\alpha=0.0$ (thermal case, dashed line) are compared with the observed data by BAT (grey points).

observed in the optical energy band: its prompt optical emission peaked at a visual magnitude of 5.3 (see Ref. 31).

We analysed the BAT prompt emission spectra integrated over different intervals of time within the fireshell model, by assuming the "modified" thermal spectrum. In Fig. 2 it is shown, as an example, the prompt emission spectrum integrated over $10 \mathrm{~s}$. It can be seen that the observational data can be correctly reproduced by assuming $\alpha=-1.8$; on the contrary, by assuming a pure thermal spectrum ( $\alpha=0.0)$ there are several discrepancies between the theoretical prediction and the observed spectrum, especially at the lower energies.

\section{Conclusion}

The analysis of various GRBs characterized by $E_{i s o} \lesssim 10^{53}$ ergs within the fireshell model has shown how the $\mathrm{N}(\mathrm{E})$ spectrum of their prompt emission can be reproduced in a satisfactory way by a convolution of thermal spectra. Nevertheless, from the study of higher energetic bursts $\left(E_{i s o} \gtrsim 10^{54} \mathrm{ergs}\right)$ such as, for example, GRB 080319B some discrepancies between the numerical simulations and the prompt emission spectra have been observed. To better explain the $\mathrm{N}(\mathrm{E})$ spectrum of the prompt emission we have introduced a phenomenologically modified thermal spectrum of photons in the comoving frame of the fireshell, whose low energy slope depends on an index $\alpha$. We have tested it by comparing the numerical simulations with the observed prompt emission spectra of GRBs. In particular we have shown, as an example, the study of GRB 080319B. From this analysis it has emerged that, by assuming a modified thermal spectrum with $\alpha=-1.8$, we can successfully reproduce the observed spectra integrated over different intervals of time, from a few seconds up to the entire prompt duration. 


\section{References}

1. J. Goodman, ApJ, 308 (1986) L47.

2. B. Paczyński, ApJ, 308 (1986) L43.

3. M. Lyutikov, R.D. Blandford, astro-ph/0312347 (2003).

4. M. Lyutikov, NJPh, 8 (2006) 119.

5. P. Mészáros, M.J. Rees, ApJ, 520 (2000) 292.

6. D. Giannios, A\&A, 457 (2006) 763.

7. F. Ryde, A. Pe'er, ApJ, 702 (2009) 1211.

8. D. Lazzati, M.C. Begelman, ApJ, 725 (2010) 1137.

9. R. Ruffini, C.L. Bianco, F. Fraschetti, ApJ, 555 (2001a) L107.

10. R. Ruffini, C.L. Bianco, F. Fraschetti, et al., ApJ, 555 (2001b) L113.

11. R. Ruffini, C.L. Bianco, P. Chardonnet et al., New perspectives in physics and astrophysics from the theoretical understanding of Gamma-Ray Bursts, in AIP Conf.Proc., eds. M. Novello and S.E. Perez Bergliaffa 668 (2003) 16.

12. R. Ruffini, M.G. Bernardini, C.L. Bianco, et al., The Blackholic energy: long and short Gamma-Ray Bursts (New perspectives in physics and astrophysics from the theoretical understanding of Gamma-Ray Bursts, II, in AIP Conf. Proc., 782 (2005a) 42 .

13. C.L. Bianco, R. Ruffini, ApJ, 605 (2004) L1.

14. C.L. Bianco, R. Ruffini, ApJ, 620 (2005b) L23.

15. R.D. Blandford, C.F. McKee, PhFL, 19 (1976) 1130.

16. P. Mészáros, P. Laguna, M.J. Rees, ApJ, 415 (1993) 181.

17. R. Sari, ApJ, 489 (1997) L37.

18. R. Sari, ApJ, 494 (1998) L49.

19. E. Waxman, ApJ, 491 (1997) L19.

20. M. J. Rees, P. Mészáros, ApJ, 496 (1998) L1.

21. Granot, J., Piran, T., Sari, R., ApJ, 513 (1999) 679.

22. A. Panaitescu, P. Mészáros, ApJ, 493 (1998) L31.

23. T. Piran, Phys. Rep., 314 (1999) 575.

24. A. Gruzinov, E. Waxman, ApJ, 511 (1999) 852.

25. J. Van Paradijs, C. Kouveliotou, R.A.M.J. Wijers, ARA\&A, 38 (2000) 379.

26. P. Mészáros, ARA $\& A, 40$ (2002) 137.

27. M. G. Bernardini, C.L. Bianco, P. Chardonnet, et al., ApJ, 634, (2005) L29.

28. R. Ruffini, R., C.L. Bianco, P. Chardonnet, et al., IJMPD, 13 (2004) 843.

29. P.M. Vreeswijk et al., GCN Circular (2008) 7444.

30. S. Golenetskii et al., GCN Circular (2008) 7482.

31. J. Racusin, S.V. Karpov, M. Sokolowski et al., Nature 455 (2008) 183. 